\title{
Smart Passive Sensors with RFID Read-Out for Item Tagging
}

Hoffmann, Martin, Schneider, Mike

University of Technology Ilmenau, Institute for Micro- and Nanotechnologies, Department

Micromechanical Systems, Max-Plank-Ring 12, 98693 IImenau

Menges, Georg

NXP Semiconductors Germany GmbH, Stresemannallee 101, 22529 Hamburg

Werner, Martin

Alcan Packaging Singen GmbH, Alusingen-Platz 1, 78221 Singen

\section{Introduction}

The integration of RFID functionality into packaging materials is a new approach for item tagging and opens new aspects as compared to bar code labels or RFID labels. The BMBF-funded project SMARTPACK that is driven by an RFID-chip manufacturer (NXP), a manufacturer of packaging material (Alcan Packaging) and a machine manufacturer (ASEM - Mühlbauer) aims for the integration of RFID functionality already during packaging material manufacturing. To allow the mass-volume production as required for item level tagging, a roll-to-roll process is targeted. Thus, the integration leads to a substantial reduction of production costs of RFID functionality by cutting down the value chain. The aim is the implementation of item level tags with extended functionality into end customer packages (fig. 1).
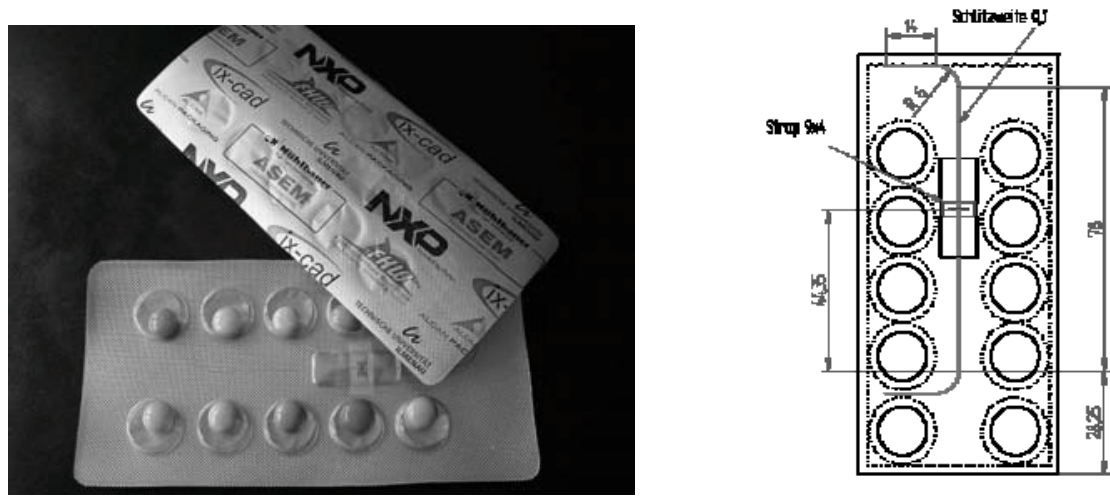

Figure 1: Blister with integrated slit antenna and RFID-chip (left); slit antenna design (right)

Besides an advanced product safety due to integrated RFID, item tagging also allows to add sensors that allow to control the product status inside a package. For cost and safety reasons complex smart active labels are not suitable for this application. New concepts for passive sensors are required that collect information on the product status during storage without permanent power supply and represent the integrity and quality at the time of read-out, preferentially as good / bad decision (fig.2).
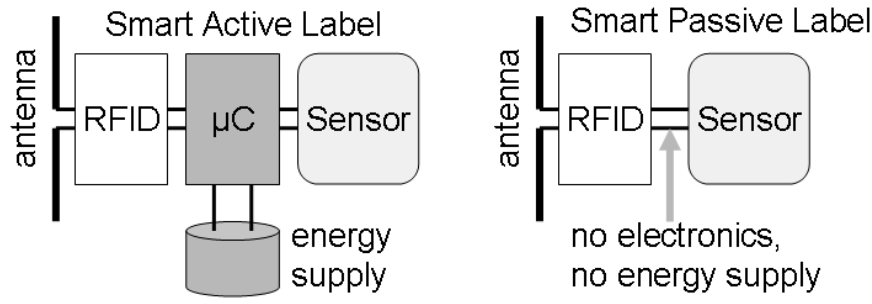

Figure 2: Active vs. Passive RFID Label concepts

For many applications, the temperature-over-time integral (TTI) is of highest interest. It is characteristic for predicting the quality of perishable goods such as fish or other food, but also to control the sterilisation process of medical goods or nonperishables: a low TTI is an indicator of insufficient sterilisation; a very high TTI indicates a degradation of vital parts. On the other hand, a sensor detecting a single overtemperature (e.g. a short transport at high temperature) is almost not required; the increase of bacteria is small whereas the increase of bacteria may be dramatical if the good is stored for long time a few degrees above the prescribed temperature. Therefore, the project SMARTPACK aimed for fully pasive TTI sensor concepts. 


\section{TTI-Concept 1: Diffusion-based Sensor for Cold Chain Management}

For perishable goods, a diffusion-based sensor is designed that changes its resistance proportional to the amount of diffused water and thus the TTI (fig. 3). The process is self-starting by filling the package with a water-containing good whereas storage in a dry ambient does not affect the sensor. A barrier layer adapted to the required TTI characteristic is used to control the diffusion into a polymer with decreasing conductivity in the presence of water. A limit switch within the RFID chip changes a bit cell in the memory if a pre-defined conductivity (e. g. $<500 \mathrm{k} \Omega$ ) is reached. During read-out of the RFID, the status of this bit cell represents the status of the TTI. For enhanced safety or a more-detailed information, two or more sensing areas can be implemented as the area per sensor is very small.

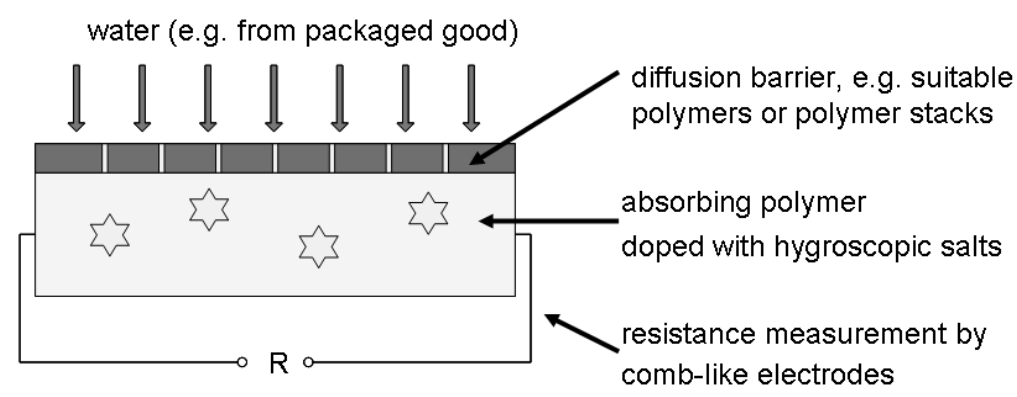

Figure 3: Diffusion-based temperature-time integrating sensor

Polymer membranes are permeable for water depending on the surrounding temperature according to a Arrhenius-ansatz [1]. The permeation coefficient is given by

$$
P=P_{0} \cdot e^{-\frac{E}{R T}} \quad \text { (equ. 1) with } P_{0}=S_{0} \cdot D_{0} \text { and } E=\Delta H_{S}+E_{D}
$$

$D_{0}$ is the diffusion coefficient, $S_{0}$ the soluability coefficent of the barrier material; R: universal gas constant; $\mathrm{T}$ : temperature; E: activation energy of the permeation process.

Integrating the amount of water permeating with $\mathrm{P}$ through the diffuision barrier results in the required information: temperature over time. This integration is done in a water-absorbing polymer that changes its resistance with the amount of absorbed water.

Therefore, the sensor consists of two layers. A polymer membrane is selected with respect to the required permeation coefficient [2]. A Nafion ${ }^{\circledR}$-based storage layer absorbes the permeating water [3, 4] and changes its conductivity proportional to its amount. Nafion ${ }^{\circledR}$ is a commercial fluorinated polymer guiding protons and water molecules. The storage layer is coated on an interdigital finger structure; changes of the volume conductivity of the storage layer thus result in a change of the resistance between the finger structure. It should be noted that for most food products an inexhaustible source of water can be assumed above the diffusion barrier.

The following fig. 4 shows the measured characteristic of a sensor consisting of the described Nafion ${ }^{\circledR}$ based storage layer and a simple photoresist coating that shows a high permeation coefficient. Without this barrier layer, the storage layer decreases its resistance heavily in a few seconds due to air humidity.

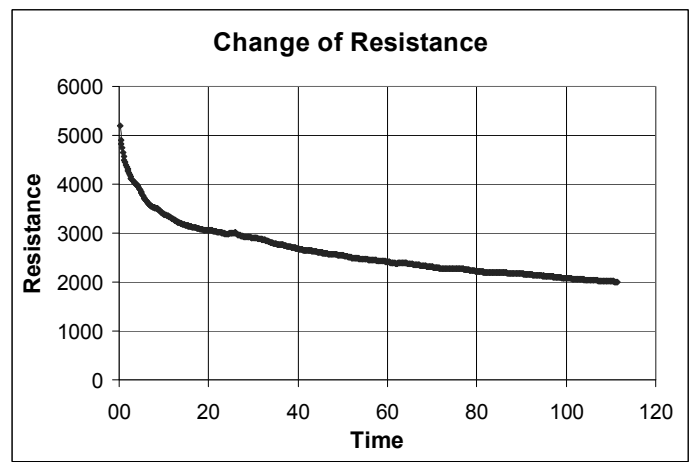

Figure 4: integration of diffusion based Sensor in Packaging 
By choosing the barrier layer, this sensor concept can easily be adapted to the required TTI-value: a smaller value for e.g. fish or poultry or a higher value for processed meat such as sausages. Further work on concrete case studies is planned for the near future.

\section{TTI-Concept 2: Capillary-Force Sensor for Sterilisation Processes}

Monitoring of sterilisation (e. g. $120^{\circ} \mathrm{C}$ and $45 \mathrm{~min}$ ) is essential for many applications, but the abovedescribed sensor is not well-suited for this application. Most barrier layers are very permeable at high temperature. A suitable alternative is a sensor based on capillary effects (fig. 4). A molten polymer fills a defined channel by capillary force. At defined positions of the channel, a set of electrodes is placed and a reservoir is filled with a suitable wax. At the required sterilisation temperature, the wax is melting and it is driven into the channel. The flow rate depends on the viscosity and thus on the temperature. After a minimum time at elevated temperature, the first electrode is reached (sterilisation has been started); a second electrode detects the optimum time and the third electrode detects exceeded heating and thus degradation. The wax based on non-toxic polymers is filled with carbon for sufficient conductivity and an increased viscosity for shortend channel length. The digital signal at the three electrodes directly indicates the status of sterilisation: minimum, good, degraded. Storage of this sensor below the wax melting point does not affect the sensor.

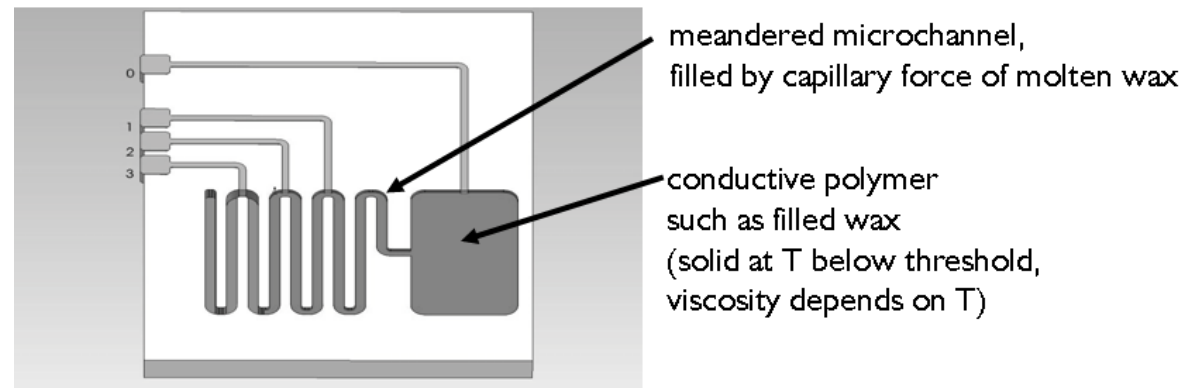

Figure 5: TTI-sensor based on capillary effects in microchannels

The principle shown in fig. 5 is based on capillary effects. A polymer i. e. wax is melting at a certain temperature (in case of sterilisation typically $120{ }^{\circ} \mathrm{C}$ ) and moves due to capillary forces into the meandering channel. The main factors on the behaviour, especially the velocity of the molten polymer, are the temperature $T$, the geometry $o$ the channel $(R, x)$ and the temperature depending viscosity (in form of shear stress $\tau$ and contact angle $\Theta_{\mathrm{Is}}$ ) of the polymeric fluid itself. These factors are displayed in the simplified Navier Stokes Equation (eq. 1) where contact angle and shear stress of the fluid are determined by experiment.) The equation is given here for the case of a vertical capillary.

$$
\frac{d}{d t}\left(m \frac{d x}{d t}\right)=-m^{\prime} g-2 \tau \pi R\left(x+x_{0}\right)+2 \pi R \sigma_{\mathrm{lg}} \cos \Theta_{l s}
$$

with $\mathrm{x}$ : direction of flow, $\mathrm{x}_{0}$ : starting point; $\mathrm{m}$ : mass of the liquid column, $\mathrm{m}^{\prime}$ : mass of the liquid column above the surface of the reservoir; $\tau$ : shear stress; R: radius of the capillary tube; $\sigma_{\mathrm{lg}}$ : surface tension between liquid and gas; $\Theta_{1 \mathrm{~s}}$ : contact angle between liquid and surface

With fixed channel geometry (and usually known time of sterilisation), the temperature integral of each package inside an autoclave can be monitored. If the fluid did not reach the second electrode, the temperature integral was too low and the product is not sterile. If the third electrode is reached, the temperature over time was too high; essential ingredients of food might be destroyed.

Based on the equation and first simulations meandering structures were modelled in order to find suitable geometries for the capillary channels. The comparison between simulation and an experiment using test channels are shown in figure 6. 


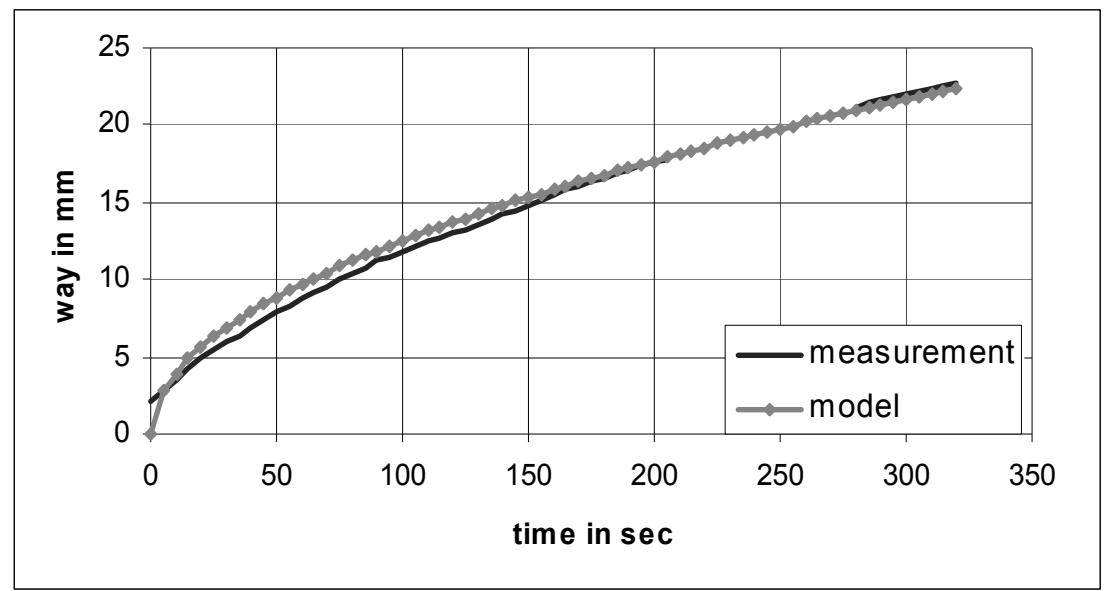

Figure 6: Comparison of mathematical model and measurement

As expected the velocity of the fluid decreases along the channel due to the increase of friction, see equation 1. The conductive fluid used for the tests is a mixture of wax and soot particles with a size of approx. $30 \mathrm{~nm}$.

Based on the first model a demonstrator was realized based on SU8 for the channels and a glass cover with gold electrode. TTI sensor samples realised by this technology are shown in fig. 7 .
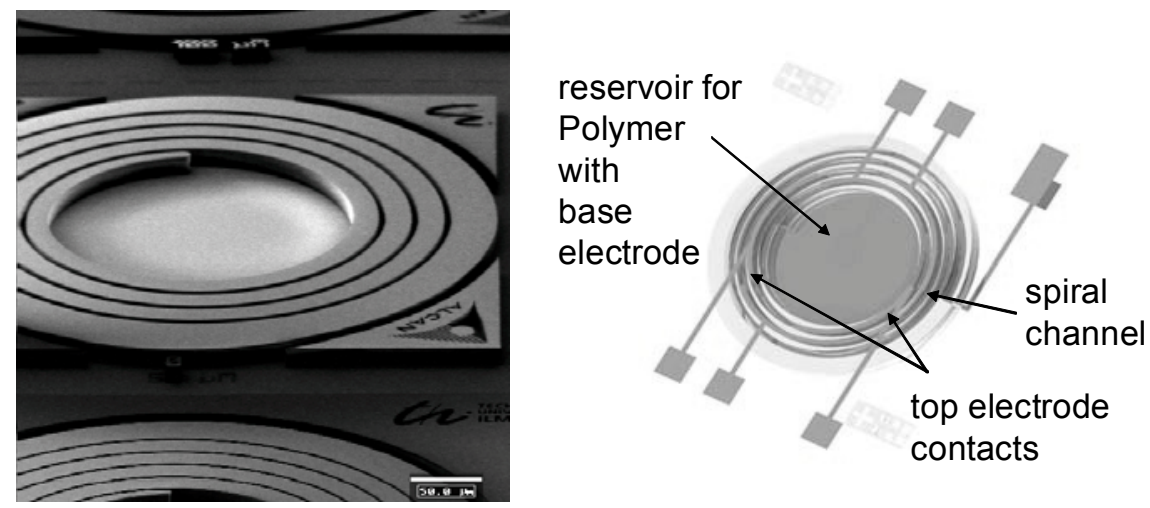

Figure 7: SEM photograph of a spiral-shaped sensor, right: 3D-sketch of the sensor

First functional samples have been fabricated by standard microsystem technologies as shown in fig. 7 . Additionally, a trial with a polymer-coated aluminium-based packing material was also performed. Fig. 8 shows the imprint of a hot embossing tool generated from the mask used for fig. 7. Hot embossing is a proven low-cost technology to achieve highly accurate structures in thermoplastic polymers.

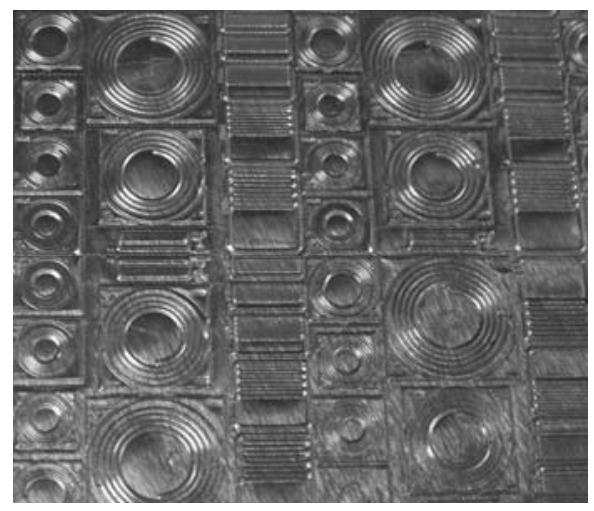

Fig. 8: hot embossed channels in a commercial packaging material 


\section{Summary:}

So-called RFID item tagging requires new concepts for the RFID integration. A successful solution is to place the RFID chip into the packaging material. As many packaging materials contain aluminium foils, it was shown that a slit antenna within this foils can replace external, much more complex labels.

This new integration technology for RFID opens the path for new applications such as integrated sensors that deliver information on the status of packaged goods. As one of the most critical parameters the temperature-over-time integral has been identified.

For supervision of cold-chain-management, a diffusion-based concept has been developed that integrates by collecting the permeating water from the good into a storage layer. The change of resistance in the storage layer is directly related to the TTI information.

For high-temperature applications such as sterilisation processes a capillary force sensor has been investigated. A molten polymer diffuses into a channel after reaching the required temperature. The length of the path that the molten polymer follows depends on the temperature (resulting in different viscosity) and the time at increased temperature.

Further fully passive sensing technologies for other relevant environmental parameters are currently under investigation. For low-cost applications close to the customer the realisation of sensor concepts without any (electrical) energy storage or energy harvesting is essential and a first step in this direction has been opened. It was also shown, that almost only materials well-known from packaging can be used for these sensors, so it is not expected that there will be environmental or health risks caused by applying these sensors in item tagging. Moreover, sensors will increase the food safety when applied to perishable goods.

\section{Acknowledgement}

The authors would like to thank the BMBF for funding the project SMARTPACK. They also thank the colleagues from NXP and Alcan Packaging Singen for many discussions concerning the requirements needed for sensors in RFID applications and the team from TU IImenau for their support in fabricating first sample demonstrators, especially Eric Markweg and Clemens Wystup.

\section{References}

$1 \quad$ R.M. Barrer, "Diffusion in and through solids", University Press, Cambridge 1941

2 S. Pauly, „Permeability and diffusion data“ in Polymer Handbook 4th ed., J. Brandrup, E.H. Immergut, E.A. Grulke (Eds.), Wiley \&Sons 1999

3 S. Nemat-Nasser and C.W. Thomas „Ionomeric Polymer-Metal Composites“ in „Electroactive Polymer actuators as artificial muscles", Y. Bar-Cohen (ed.), SPIE Press 2001

$4 \quad$ K. Broka and P. Ekdunge, J. Appl. Electrochem. 27(1997), 117-23

5 N.-T. Nguyen, Mikrofluidik - Entwurf, Herstellung und Charaktierisierung, Teubner Verlag, 2004 